\title{
Eigenvalues and eigenvectors of the transfer matrix involved in the calculation of geomagnetically induced currents in an electric power transmission network
}

\author{
Risto Pirjola \\ Finnish Meteorological Institute, P. O. Box 503, FI-00101 Helsinki, Finland \\ (Received February 21, 2011; Revised June 9, 2011; Accepted June 9, 2011; Online published January 26, 2012)
}

\begin{abstract}
Geomagnetically induced currents (GIC) owing in power grids can be calculated from matrix equations whose input data are the geoelectric eld and the network parameters. The transfer matrix between the "perfect-earthing" (pe) currents and the earthing GIC are discussed in this paper by considering its eigenvalues and eigenvectors. The pe currents include the in uence of the geoelectric eld whereas the transfer matrix only depends on the network data. It is shown that an eigenvalue equals one or the corresponding eigenvector satis es the condition that the sum of its pe currents is zero. Using physical arguments, we conclude that all eigenvalues of the transfer matrix are non-negative and between zero and one. This statement is proved mathematically for a three-node network and supported by numerical computations for the Finnish $400 \mathrm{kV}$ GIC test model. Special attention is paid to the norm of the earthing GIC, which gives an idea of the risk of GIC to a power grid. This norm seems to have the lower and upper limits practically equal to the smallest and largest $(\neq 1)$ eigenvalue of the transfer matrix multiplied by the norm of the pe currents.
\end{abstract}

Key words: Geomagnetically induced current, GIC, power grid, space weather, transfer matrix, eigenvalue, norm, scalar product.

\section{Introduction}

Geomagnetically induced currents (GIC) are ground effects of space weather, whose origin is in solar activity. When owing in electric power transmission networks, oil and gas pipelines, telecommunication cables or railway circuits, GIC may cause problems to the particular system. The history of GIC dates back to the rst telegraph systems in the mid-1800's (e.g. Boteler et al., 1998; Lanzerotti et al., 1999). In power grids, the problems result from transformer saturation due to GIC (e.g. Bolduc, 2002; Molinski, 2002; Kappenman, 2007). In the worst cases, wide-spread blackouts and permanent damage of transformers may occur.

GIC in a power network can be investigated by measurements or by theoretical calculations. GIC values vary spatially much from site to site in a network. Therefore GIC data recorded at one place does not provide information about GIC values at other locations. Thus in practice, model calculations are needed to obtain a full overall understanding of GIC magnitudes and of the resulting risks and threats to a power grid. The calculations can be veri ed and adjusted by GIC measurements at one or a few sites.

A GIC calculation consists of two separate parts (e.g. Pirjola, 2002): (1) Determination of the horizontal geoelectric eld at the Earth's surface (called the "geophysical

Copyright (C) The Society of Geomagnetism and Earth, Planetary and Space Sciences (SGEPSS); The Seismological Society of Japan; The Volcanological Society of Japan; The Geodetic Society of Japan; The Japanese Society for Planetary Sciences; TERRAPUB.

doi:10.5047/eps.2011.06.017 part"), and (2) Computation of GIC produced by the horizontal geoelectric eld in the network (called the "engineering part"). The geophysical part, which is independent of the network considered, may be solved by using Maxwell's equations and the boundary conditions of the electric and magnetic elds. The input consists of information or assumptions about the ground conductivity and about the currents owing in the ionosphere and magnetosphere or about the geomagnetic variations at the Earth's surface. Practical techniques to be applied to the geophysical part are discussed, for example, by Viljanen et al. (2004).

Besides the geoelectric eld, the network topology and resistances must be known for solving the engineering part. Time variations of the geomagnetic and geoelectric elds and of GIC are very slow compared to the $50 / 60 \mathrm{~Hz}$ frequency used in power transmission. Therefore, a dc treatment is applicable to the engineering part. Lehtinen and Pirjola (1985) present a matrix method for determining the earthing GIC owing between a power grid and the Earth as well as GIC in transmission lines. The basic equation expresses the earthing GIC as a column matrix in terms of another column matrix that consists of so-called "perfectearthing" currents. They involve the dependence on the geovoltages accompanying the horizontal geoelectric eld. To the author's knowledge, the properties of the transfer matrix between the two column matrices have not been discussed in the GIC literature except for a brief study by Pirjola (2009). In this paper, we go deeper into this investigation with the aim to be able to draw physical conclusions from the eigenvalues and eigenvectors of the transfer 
matrix. The whole subject of matrices and their eigenvalues and eigenvectors refers mathematically to linear vector spaces. In this paper, however, discussions about abstract mathematical concepts and details are minimised.

The technique presented by Lehtinen and Pirjola (1985) is summarised in Section 2 including the de nition of the transfer matrix in terms of network parameters. Eigenvalues and eigenvectors of the transfer matrix are discussed in Section 3. We consider the case of a three-node network in detail because it is simple enough to allow exact calculations and equations in practice. It is evident that conclusions can be drawn from the three-node case that might be extended and generalised to also hold true for more complex networks. Other numerical results presented in Section 3 concern the Finnish $400 \mathrm{kV}$ network, which is introduced as a GIC test model by Pirjola (2009). In Section 3, special attention is paid to the norm of the earthing GIC column matrix, which provides a measure of GIC impacts on the system. Concluding remarks are given in Section 4.

\section{Calculation of Geomagnetically Induced Cur- rents in a Power Network}

A horizontal geoelectric eld $\boldsymbol{E}$ impacting a network of conductors with $N$ discrete nodes, called stations and earthed by the resistances $R_{e, i}(i=1, \ldots, N)$, implies the ow of geomagnetically induced currents (GIC) in the network and between the network and the Earth. Lehtinen and Pirjola (1985) derive a formula for the $N \times 1$ column matrix $\boldsymbol{I}_{e}$ that includes the earthing currents (or earthing GIC) $I_{e, m}(m=1, \ldots, N)$ to (positive) or from (negative) the Earth at the stations as follows

$$
\boldsymbol{I}_{e}=\left(\mathbf{1}+\boldsymbol{Y}_{n} \boldsymbol{Z}_{e}\right)^{-1} \boldsymbol{J}_{e}
$$

The symbol 1 denotes the $N \times N$ unit identity matrix. The $N \times N$ earthing impedance matrix $\boldsymbol{Z}_{e}$ and the $N \times N$ network admittance matrix $\boldsymbol{Y}_{n}$, as well as the $N \times 1$ column matrix $\boldsymbol{J}_{e}$ are explained below.

The de nition of $\boldsymbol{Z}_{e}$ states that multiplying the earthing current matrix $\boldsymbol{I}_{e}$ by $\boldsymbol{Z}_{e}$ gives the voltages between the earthing points and a remote Earth that are related to the ow of the currents $I_{e, m}(m=1, \ldots, N)$. Thus, expressing the voltages by an $N \times 1$ column matrix $\boldsymbol{U}$, we have

$$
\boldsymbol{U}=\boldsymbol{Z}_{e} \boldsymbol{I}_{e}
$$

Utilising the reciprocity theorem, $\boldsymbol{Z}_{e}$ can be shown to be a symmetric matrix. The diagonal elements of $\boldsymbol{Z}_{e}$ equal the earthing resistances of the stations. If the distances of the stations are large enough, the in uence of the current $I_{e, m}$ at one station on the voltages at other stations is negligible, and then the off-diagonal elements of $\boldsymbol{Z}_{e}$ are zero (see Pirjola, 2008).

The matrix $\boldsymbol{Y}_{n}$ is de ned by

$$
\begin{aligned}
& (i \neq m): Y_{n, i m}=-\frac{1}{R_{n, i m}}, \\
& (i=m): Y_{n, i m}=\sum_{k=1, k \neq i}^{N} \frac{1}{R_{n, i k}}
\end{aligned}
$$

where $R_{n, i m}$ is the resistance of the conductor between stations $i$ and $m(i, m=1, \ldots, N)$. (If stations $i$ and $m$ are not directly connected by a conductor, $R_{n, i m}$ naturally gets an in nite value.) It is directly seen from Eq. (3) that $\boldsymbol{Y}_{n}$ is symmetric.

The elements $J_{e, m}(m=1, \ldots, N)$ of the column matrix $J_{e}$ are given by

$$
J_{e, m}=\sum_{i=1, i \neq m}^{N} \frac{V_{i m}}{R_{n, i m}}
$$

The geovoltage $V_{i m}$ is produced by the horizontal geoelectric eld $\boldsymbol{E}$ along the path de ned by the conductor line from station $i$ to station $m(i, m=1, \ldots, N)$, i.e.

$$
V_{i m}=\int_{i}^{m} \boldsymbol{E} \cdot d \boldsymbol{s}
$$

Generally, the geoelectric eld is rotational, and so the integral in Eq. (5) is path-dependent. Thus, as indicated, the integration route must follow the conductor between $i$ and $m$ (see Boteler and Pirjola, 1998). Equations (4) and (5) show that the column matrix $\boldsymbol{J}_{e}$ involves the contribution of the geoelectric eld to Eq. (1). Since $\boldsymbol{I}_{e}$ and $\boldsymbol{J}_{e}$ are equal in the case of perfect earthings, i.e. when $\boldsymbol{Z}_{e}=0$, the elements of $\boldsymbol{J}_{e}$ are called "perfect-earthing" ( $p e$ ) earthing currents.

When GIC in a power network are calculated, it is convenient to treat the three phases as one circuit element. The resistance of the element is then one third of that of a single phase, and the GIC owing in the element is three times the current in a single phase. Moreover, the earthing resistances (which might be called the total earthing resistances) are assumed to include the actual earthing resistances, the transformer resistances and the resistances of possible reactors or any resistors in the earthing leads of transformer neutrals.

Regarding the analysis included in this paper, it is useful to note that Lehtinen and Pirjola (1985) also present another derivation and interpretation of Eq. (1) by neglecting the geoelectric eld and assuming an external injection of the pe currents $J_{e, m}$ into the nodes $(m=1, \ldots, N)$. Although Lehtinen and Pirjola (1985) do not express it explicitly, it seems obvious that the ctitious injection corresponds to the use of so-called Norton's equivalent current sources (see also Pirjola, 2009). Physical reasons require that

$$
\sum_{m=1}^{N} J_{e, m}=0
$$

Equation (6) means that the sums of the pe currents owing to and from the network are equal. De ning $J_{e, m}$ by formula (4), Eq. (6) is automatically satis ed, which is a consequence from $V_{i m}=-V_{m i}$ and $R_{n, i m}=R_{n, m i}$. (Equation (6) also holds true for the $I_{e, m}$ currents obtained from formula (1).)

It should be noted that knowing the currents $J_{e, m}$ and the resistances $R_{n, i m}$ does generally not determine the voltages $V_{i m}$ uniquely $(i, m=1, \ldots, N)$ from Eq. (4). This can easily be seen, for example, by considering a simple threenode system in which all three line resistances are equal to $R_{n, 12}=R_{n, 23}=R_{n, 31}=1 \Omega$. The two different voltage sets $\left(V_{12}=0 \mathrm{~V}, V_{23}=-200 \mathrm{~V}, V_{31}=100 \mathrm{~V}\right)$ and $\left(V_{12}=100 \mathrm{~V}, V_{23}=-100 \mathrm{~V}, V_{31}=200 \mathrm{~V}\right)$ give the 
same currents $J_{e, 1}=100 \mathrm{~A}, J_{e, 2}=200 \mathrm{~A}$ and $J_{e, 3}=-300$ A.

\section{Eigenvalues and Eigenvectors of the Transfer Matrix $C=\left(1+Y_{n} Z_{e}\right)^{-1}$}

\subsection{General situation}

We denote the $N \times N$ transfer matrix $\left(\mathbf{1}+\boldsymbol{Y}_{n} \boldsymbol{Z}_{e}\right)^{-1}$ between $\boldsymbol{J}_{e}$ and $\boldsymbol{I}_{e}$ appearing in Eq. (1) by $\boldsymbol{C}$. Pirjola (2009) shows that the sum of the elements $C_{m i}(i, m=1, \ldots, N)$ in every column of $\boldsymbol{C}$ equals one, i.e.

$$
\sum_{m=1}^{N} C_{m i}=1 \quad(i=1, \ldots, N)
$$

Let $\lambda$ and $\boldsymbol{J}$ (neglecting the subscript ' $e$ ' from now on in this paper) be an eigenvalue and eigenvector of $\boldsymbol{C}$, respectively. Then $\boldsymbol{J}$ is an $N \times 1$ column matrix with the elements $J_{m}(m=1, \ldots, N)$ that satis es

$$
\boldsymbol{C J}=\lambda \boldsymbol{J}
$$

or in the element form

$$
\sum_{i=1}^{N} C_{m i} J_{i}=\lambda J_{m} \quad(m=1, \ldots, N)
$$

A simple basic property of all matrices is that if $\lambda$ is an eigenvalue, $\boldsymbol{J}$ the corresponding eigenvector and $\beta$ an arbitrary scalar, then $\beta \boldsymbol{J}$ is also an eigenvector of the same matrix associated with the same eigenvalue $\lambda$.

Summing the right and left sides of Eq. (9) over $m$ from 1 to $N$, changing the order of the sums on the left side and using Eq. (7) easily give

$$
(1-\lambda) \sum_{k=1}^{N} J_{k}=0
$$

Thus always, either the eigenvalue $\lambda$ equals one or the eigenvector $\boldsymbol{J}$ satis es the physical condition included in Eq. (6).

Based on Eqs. (1) (with the neglect of the subscript ' $e$ ') and (8), we immediately see that if a $(\lambda, \boldsymbol{J})$ pair is an eigenvalue and an eigenvector of the matrix $\boldsymbol{C}$, then the corresponding earthing current $N \times 1$ column matrix is

$$
\boldsymbol{I}=\lambda \boldsymbol{J}
$$

This means that the pe earthing currents constituting the column matrix $\boldsymbol{J}$ are changed by a coef cient $\lambda$ to give the earthing currents $I_{m}(m=1, \ldots, N)$ included in the column matrix $\boldsymbol{I}$ and appearing when the correct non-zero earthing resistances are taken into account. Considering the interpretation that the $p e$ currents are externally injected into the nodes, the case $\lambda=1$ clearly simply means that at each node the injected current directly ows into the Earth. This is not an interesting situation from the point of view of GIC research, and in particular, as pointed out, this case does not presume the physical equation (6) to be satis ed.

A natural assumption is that $I_{m}$ and $J_{m}$ ow in the same direction, so that $\lambda \geq 0$. It seems obvious that the earthing currents $I_{m}$ cannot be larger than the corresponding pe currents $J_{m}$, and so $\lambda \leq 1$. Consequently, based on these physical arguments, the eigenvalues $\lambda$ of the matrix $\boldsymbol{C}$ are expected to satisfy the inequalities

$$
0 \leq \lambda \leq 1
$$

We can de ne the magnitude of the earthing currents, which may also be called the absolute value or the norm of the earthing currents, as

$$
|\boldsymbol{I}|=\sqrt{\sum_{k=1}^{N}\left(I_{k}\right)^{2}}
$$

The norm of the $p e$ currents $(=|\boldsymbol{J}|)$ is naturally de ned with a similar formula. If $\boldsymbol{J}$ is an eigenvector of $\boldsymbol{C}$ with the eigenvalue $\lambda$, Eq. (11), together with the inequalities (12), shows that $|\boldsymbol{I}| \leq|\boldsymbol{J}|$, and normalising the column matrix $\boldsymbol{J}$ to unity, i.e. $|\boldsymbol{J}|=1,|\boldsymbol{I}|$ equals $\lambda$. It is worth noting that the unit of $\boldsymbol{I}$ and $\boldsymbol{J}$ is [A] whereas $\lambda$ is dimensionless. Thus, to be precise, we should actually say that $|\boldsymbol{J}|=1 \mathrm{~A}$ and $|\boldsymbol{I}|$ equals $\lambda \mathrm{A}$. In this paper, however, no confusion can arise even if the use of the units is somewhat inaccurate.

\subsection{Three-node network}

3.2.1 Arbitrary resistances Now we consider a three-node network (i.e. $N=3$ ), which is simple enough to enable analytic calculations, but it does not lead to trivial conclusions as a two-node system would do. Let us number the nodes by 1, 2 and 3 and assume that their earthing resistances are $S_{1}, S_{2}$ and $S_{3}$, respectively. The line resistance between the nodes 1 and 2 is denoted by $R_{1}$, between the nodes 2 and 3 by $R_{2}$ and between the nodes 3 and 1 by $R_{3}$. (Thus, compared to Section 2, the notation is simplied by omitting the subscripts ' $e$ ' and ' $n$ ' and by using the symbol ' $S$ ' distinguishing the earthing resistances from the line resistances more clearly. The line resistances also have only one subscript now.) We assume that the nodes are sufciently distant to permit the assumption that the earthing impedance matrix $\boldsymbol{Z}_{e}$ is diagonal with $S_{1}, S_{2}$ and $S_{3}$ being the diagonal elements. Pirjola (2008) indicates that this is generally not a serious or critical limitation in practical GIC studies.

Utilising the de nition of the matrix $\boldsymbol{C}$ given in the beginning of Section 3.1 and Eq. (3) and performing basic (but somewhat tedious) matrix calculations, the following equation is obtained

$$
\boldsymbol{C}=\frac{1}{F}\left(\begin{array}{ccc}
1+A_{1}+B_{1} & \alpha_{21}+B_{1} & \alpha_{33}+B_{1} \\
\alpha_{11}+B_{2} & 1+A_{2}+B_{2} & \alpha_{32}+B_{2} \\
\alpha_{13}+B_{3} & \alpha_{22}+B_{3} & 1+A_{3}+B_{3}
\end{array}\right)
$$

where

$$
\begin{aligned}
& \alpha_{j k}=\frac{S_{j}}{R_{k}} \quad(j, k=1,2,3) \\
& A_{1}=\alpha_{21}+\alpha_{22}+\alpha_{32}+\alpha_{33} \\
& A_{2}=\alpha_{11}+\alpha_{13}+\alpha_{32}+\alpha_{33} \\
& A_{3}=\alpha_{11}+\alpha_{13}+\alpha_{21}+\alpha_{22}
\end{aligned}
$$




$$
\begin{gathered}
B_{1}=\alpha_{21} \alpha_{32}+\alpha_{21} \alpha_{33}+\alpha_{22} \alpha_{33} \\
B_{2}=\alpha_{11} \alpha_{32}+\alpha_{11} \alpha_{33}+\alpha_{13} \alpha_{32} \\
B_{3}=\alpha_{11} \alpha_{22}+\alpha_{13} \alpha_{21}+\alpha_{13} \alpha_{22} \\
F=\operatorname{det}\left(\boldsymbol{C}^{-1}\right)=\operatorname{det}\left(\mathbf{1}+\boldsymbol{Y}_{n} \boldsymbol{Z}_{e}\right) \\
=1+\alpha_{11}+\alpha_{13}+\alpha_{21}+\alpha_{22}+\alpha_{32}+\alpha_{33}+\alpha_{11} \alpha_{22} \\
+\alpha_{11} \alpha_{32}+\alpha_{11} \alpha_{33}+\alpha_{13} \alpha_{21}+\alpha_{13} \alpha_{22}+\alpha_{13} \alpha_{32} \\
+\alpha_{21} \alpha_{32}+\alpha_{21} \alpha_{33}+\alpha_{22} \alpha_{33} \\
=1+\frac{1}{2}\left(A_{1}+A_{2}+A_{3}\right)+B_{1}+B_{2}+B_{3}
\end{gathered}
$$

and 'det' stands for the determinant of a matrix. (Note that it follows from Eq. (18) that $F$ equals the inverse value of $\operatorname{det}(\boldsymbol{C}))$.

The eigenvalues $\lambda$ of $\boldsymbol{C}$ satisfy the equation

$$
\operatorname{det}(\boldsymbol{C}-\lambda \mathbf{1})=0
$$

which is a polynomial equation of the third degree for $\lambda$ in this particular case. After long and tedious algebraic manipulations, Eq. (19), together with formulas (14)-(18), leads to the following equation

$$
(\lambda-1)\left(F \lambda^{2}-(2+L) \lambda+1\right)=0
$$

where

$$
\begin{aligned}
L & =\alpha_{11}+\alpha_{13}+\alpha_{21}+\alpha_{22}+\alpha_{32}+\alpha_{33} \\
& =\frac{1}{2}\left(A_{1}+A_{2}+A_{3}\right)
\end{aligned}
$$

De ning

$$
\begin{aligned}
P= & \alpha_{11} \alpha_{22}+\alpha_{11} \alpha_{32}+\alpha_{11} \alpha_{33}+\alpha_{13} \alpha_{21}+\alpha_{13} \alpha_{22} \\
& +\alpha_{13} \alpha_{32}+\alpha_{21} \alpha_{32}+\alpha_{21} \alpha_{33}+\alpha_{22} \alpha_{33} \\
= & B_{1}+B_{2}+B_{3}
\end{aligned}
$$

the determinant $F$ can be written as

$$
F=1+L+P
$$

Equation (20) directly gives the three eigenvalues of $\boldsymbol{C}$ as follows

$$
\begin{aligned}
& \lambda_{1}=1 \\
& \lambda_{2}=\frac{2+L+\sqrt{(2+L)^{2}-4 F}}{2 F} \\
& \lambda_{3}=\frac{2+L-\sqrt{(2+L)^{2}-4 F}}{2 F}
\end{aligned}
$$

As mentioned in Section 3.1, the case of an eigenvalue equal to one $\left(\lambda_{1}=1\right)$ is less interesting regarding GIC research. Thus our discussion is concentrated on the eigenvalues $\lambda_{2}$ and $\lambda_{3}$. The discriminant

$$
\begin{aligned}
D= & (2+L)^{2}-4 F=L^{2}-4 P \\
= & \left(\alpha_{11}+\alpha_{13}+\alpha_{21}-\alpha_{22}-\alpha_{32}-\alpha_{33}\right)^{2} \\
& -4\left(\alpha_{13} \alpha_{21}-\alpha_{13} \alpha_{33}-\alpha_{21} \alpha_{22}+\alpha_{22} \alpha_{33}\right)
\end{aligned}
$$

appearing in formulas (24b) and (24c) can be considered as a function of six variables $\alpha_{11}, \alpha_{13}, \alpha_{21}, \alpha_{22}, \alpha_{32}$ and $\alpha_{33}$. At the extrema of $D$, all derivatives $\frac{\partial D}{\partial \alpha_{i k}}$ are equal to zero with $(j, k)=(1,1),(1,3),(2,1),(2,2),(3,2),(3,3)$. This results in the conditions

$$
\begin{aligned}
& \alpha_{21}=\alpha_{33} \\
& \alpha_{13}=\alpha_{22} \\
& \alpha_{11}=\alpha_{32}
\end{aligned}
$$

Because $D=D\left(\alpha_{11}, \alpha_{13}, \alpha_{21}, \alpha_{22}, \alpha_{32}, \alpha_{33}\right)$ can achieve arbitrarily large positive values, the zero values of the derivatives $\frac{\partial D}{\partial \alpha_{j k}}$ are necessarily associated with minima. If Eq. (26) are satis ed, $D$ is equal to zero as can directly be seen from formula (25). Consequently, always $D \geq 0$, which proves that all eigenvalues of $\boldsymbol{C}$ are real. Since $\sqrt{(2+L)^{2}-4 F} \leq 2+L$, it follows from Eq. (24) that all eigenvalues are non-negative. Starting from the fact that $P \geq 0$ and utilising formula (23), it can be shown in a straightforward way that $2 F \geq 2+L+\sqrt{(2+L)^{2}-4 F}$, from which, based on Eq. (24), we may conclude that all eigenvalues are less than or equal to one. Consequently, in the case of a three-node network, a mathematical proof is provided for the inequalities (12), which are concluded with physical arguments in the general situation discussed in Section 3.1.

The eigenvector $\boldsymbol{J}^{k}=\left(\begin{array}{c}J_{1}^{k} \\ J_{2}^{k} \\ J_{3}^{k}\end{array}\right)$ accompanying the eigenvalue $\lambda_{k}$ is calculated from Eq. (8) in the form $\boldsymbol{C} \boldsymbol{J}^{k}=\lambda_{k} \boldsymbol{J}^{k}$ with $C$ and $\lambda_{k}$ according to Eqs. (14) and (24), respectively $(k=1,2,3)$. The result is

$J_{2}^{k}=\frac{\left(\alpha_{11}+B_{2}\right)\left(\alpha_{33}+B_{1}\right)-\left(1+A_{1}+B_{1}-\lambda_{k} F\right)\left(\alpha_{32}+B_{2}\right)}{\left(\alpha_{21}+B_{1}\right)\left(\alpha_{32}+B_{2}\right)-\left(1+A_{2}+B_{2}-\lambda_{k} F\right)\left(\alpha_{33}+B_{1}\right)} J_{1}^{k}$

$J_{3}^{k}=\frac{\left(1+A_{1}+B_{1}-\lambda_{k} F\right)\left(1+A_{2}+B_{2}-\lambda_{k} F\right)-\left(\alpha_{11}+B_{2}\right)\left(\alpha_{21}+B_{1}\right)}{\left(\alpha_{21}+B_{1}\right)\left(\alpha_{32}+B_{2}\right)-\left(1+A_{2}+B_{2}-\lambda_{k} F\right)\left(\alpha_{33}+B_{1}\right)} J_{1}^{k}$

The fact that no unique solution is obtained for all elements $J_{1}^{k}, J_{2}^{k}, J_{3}^{k}$, but $J_{2}^{k}$ and $J_{3}^{k}$ depend on $J_{1}^{k}$, which remains arbitrary, is in agreement with the statement about $\beta \boldsymbol{J}$ being also an eigenvector in Section 3.1. Mathematically this is a consequence of Eq. (19). By using Eq. (27) and doing some algebraic work, it is possible to show that the sum $J_{1}^{k}+J_{2}^{k}+J_{3}^{k}$ is proportional to $F \lambda_{k}^{2}-(2+L) \lambda_{k}+1$. Thus, based on Eq. (20), the sum is zero when $\lambda_{k} \neq 1$, which means that we have explicitly shown that Eq. (6) holds true for the eigenvectors $\boldsymbol{J}^{2}$ and $\boldsymbol{J}^{3}$ in an arbitrary three-node network.

3.2.2 Resonance case Let us investigate the situation de ned by Eq. (26) called the resonance case. By using the de nition of the $\alpha_{j k}$ quantities given by Eq. (15), we see that Eq. (26) are equivalent with

$$
S_{1} R_{2}=S_{2} R_{3}=S_{3} R_{1}
$$

Taking into account the de nition of the $S_{j}$ and $R_{j}$ resistances $(j=1,2,3)$ in the beginning of Section 3.2.1, 
Eq. (28) mean that the products of an earthing resistance and the opposite line resistance are equal in the resonance case.

Because the discriminant $D$ involved in Eqs. (24b) and $(24 c)$ is zero when Eqs. (26) and (28) are satis ed, the eigenvalues $\lambda_{2}$ and $\lambda_{3}$ are equal in the resonance case. It can be easily shown that Eq. (26) reduce the different quantities presented in Section 3.2.1 as follows

$$
\begin{aligned}
L & =2\left(\alpha_{11}+\alpha_{22}+\alpha_{33}\right) \\
P & =\frac{L^{2}}{4} \\
F & =\left(1+\frac{L}{2}\right)^{2} \\
\lambda_{2} & =\lambda_{3}=\frac{1}{1+\frac{L}{2}} \\
\boldsymbol{C} & =\frac{1}{1+\frac{L}{2}}\left(\mathbf{1}+\left(\begin{array}{lll}
\alpha_{33} & \alpha_{33} & \alpha_{33} \\
\alpha_{11} & \alpha_{11} & \alpha_{11} \\
\alpha_{22} & \alpha_{22} & \alpha_{22}
\end{array}\right)\right)
\end{aligned}
$$

where $\mathbf{1}$ in is the a $3 \times 3$ unit identity matrix.

Let $\boldsymbol{J}=\left(\begin{array}{l}J_{1} \\ J_{2} \\ J_{3}\end{array}\right)$ be an arbitrary pe current $3 \times 1$ column matrix to be used in Eq. (1). Utilising Eq. (29e), the corresponding earthing current $3 \times 1$ column matrix is

$$
\boldsymbol{I}=\boldsymbol{C} \boldsymbol{J}=\frac{1}{1+\frac{L}{2}}\left(\boldsymbol{J}+\left(J_{1}+J_{2}+J_{3}\right)\left(\begin{array}{c}
\alpha_{33} \\
\alpha_{11} \\
\alpha_{22}
\end{array}\right)\right)
$$

We see from Eq. (30) that any matrix $\boldsymbol{J}=\left(\begin{array}{l}J_{1} \\ J_{2} \\ J_{3}\end{array}\right)$ is an eigenvector of $C$ with the eigenvalue $\left(1+\frac{L}{2}\right)^{-1}$ if $J_{1}+J_{2}+$ $J_{3}=0$, or in other words, all pe current $3 \times 1$ column matrices satisfying the physical equation (6) are eigenvectors of the transfer matrix $\boldsymbol{C}$ associated with the eigenvalue $\lambda_{2}=\lambda_{3}=\left(1+\frac{L}{2}\right)^{-1}=\left(1+\alpha_{11}+\alpha_{22}+\alpha_{33}\right)^{-1}$ (Eqs. (29a) and (29d)). It is also possible to conclude from Eq. (30) with Eq. (29a) that a matrix $\boldsymbol{J}$ that satis es the formulas $J_{2}^{1}=\frac{\alpha_{11}}{\alpha_{33}} J_{1}^{1}$ and $J_{3}^{1}=\frac{\alpha_{22}}{\alpha_{33}} J_{1}^{1}$ is an eigenvector corresponding to the eigenvalue $\lambda_{1}=1$ (note the superscript " 1 " in the elements of the eigenvector). Using the de nition of the $\alpha_{j k}$ quantities and the resonance conditions (26), we obtain the relations $\frac{J_{2}^{1}}{J_{1}^{1}}=\frac{S_{1}}{S_{2}}=\frac{R_{3}}{R_{2}}$ and $\frac{J_{3}^{1}}{J_{1}^{1}}=\frac{S_{1}}{S_{3}}=\frac{R_{1}}{R_{2}}$, which indicate that the pe currents connected with the eigenvalue $\lambda_{1}=1$ are inversely proportional to the corresponding earthing resistances and proportional to the resistances of the opposite lines. These formulas further show, for example, that $J_{1}^{1}+J_{2}^{1}+J_{3}^{1}=J_{1}^{1}\left(1+\frac{S_{1}}{S_{2}}+\frac{S_{1}}{S_{3}}\right)=J_{1}^{1}\left(1+\frac{R_{3}+R_{1}}{R_{2}}\right) \neq 0$ (when $\boldsymbol{J} \neq 0$ ), so that the physical condition (6) is not satis ed.

We may also write the eigenvalue $\lambda_{2}=\lambda_{3}$ in the follow- ing ways

$$
\begin{aligned}
\lambda_{2} & =\lambda_{3}=\frac{1}{1+\frac{S_{1}}{R_{1}}+\frac{S_{2}}{R_{2}}+\frac{S_{3}}{R_{3}}} \\
& =\frac{1}{1+\frac{S_{1}}{R_{3}}+\frac{S_{3}}{R_{2}}+\frac{S_{2}}{R_{1}}}
\end{aligned}
$$

The physical contents of the resistance ratios in the last two expressions are that the earthing resistances of the nodes are divided by the resistance of the line next to this particular node. In the rst and second form, we take the next line in the direction Node $1 \rightarrow$ Node $2 \rightarrow$ Node $3 \rightarrow$ Node 1 and Node $1 \rightarrow$ Node $3 \rightarrow$ Node $2 \rightarrow$ Node 1 , respectively. Other expressions for $\lambda_{2}$ and $\lambda_{3}$ can also be derived, as for example $\lambda_{2}=\lambda_{3}=(1+g f)^{-1}$ where $g=S_{1} R_{2}=$ $S_{2} R_{3}=S_{3} R_{1}$ (see Eq. (28)) and $f=\frac{R_{1}+R_{2}+R_{3}}{R_{1} R_{2} R_{3}}$.

A special situation of the resonance case is obtained if we assume that the earthing resistances are equal $\left(S_{1}=S_{2}=\right.$ $\left.S_{3}\right)$ and the line resistances are equal $\left(R_{1}=R_{2}=R_{3}\right)$. Then all $\alpha_{j k}$ quantities are the same $(=\alpha)$. By utilising the equations mentioned above in this section, we directly see, for example, that the eigenvalues are $\lambda_{1}=1, \lambda_{2}=\lambda_{3}=$ $\frac{1}{1+3 \alpha}$ and that the elements of the eigenvector associated with $\lambda_{1}$ are equal $\left(J_{1}^{1}=J_{2}^{1}=J_{3}^{1}\right)$.

3.2.3 Numerical results Let us assume that the values of the earthing and line resistances of a three-node network are $S_{1}=1.00 \Omega, S_{2}=0.64 \Omega, S_{3}=0.88$ $\Omega, R_{1}=0.50 \Omega, R_{2}=1.20 \Omega$ and $R_{3}=0.87 \Omega$. These values are taken somewhat arbitrarily but they represent typical values for a power network (Pirjola, 2009). Figure 1(a) presents the norm, given by Eq. (13), of the earthing currents obtained from $\boldsymbol{I}=\boldsymbol{C J}$, in which it is assumed that the pe column matrix $J=\left(\begin{array}{l}J_{1} \\ J_{2} \\ J_{3}\end{array}\right)$ is normalised to unity, i.e. $|\boldsymbol{J}|=\sqrt{\left(J_{1}\right)^{2}+\left(J_{2}\right)^{2}+\left(J_{3}\right)^{2}}=1$, and that $J_{1}+J_{2}+J_{3}=0$, i.e. Eq. (6) holds true. As the input for Fig. 1(a), altogether 32660 different column matrices $J$ that satisfy these conditions are used by changing $\boldsymbol{J}$ slightly and systematically between the runs of the calculation program. Figure 1(b) depicts the scalar product values between $\boldsymbol{J}$ and a xed reference column matrix $\boldsymbol{J}_{\text {ref }}=\left(\begin{array}{c}J_{\text {ref_1 }} \\ J_{\text {ref_2 }} \\ J_{\text {ref_3 }}\end{array}\right)$ also normalised to unity and satisfying Eq. (6). The scalar product is de ned in the usual way as

$$
\left\langle\boldsymbol{J} \mid \boldsymbol{J}_{\text {ref }}\right\rangle=\sum_{k=1}^{N} J_{k} J_{\text {ref } \_k}
$$

In the present case $N$ equals 3, but Eq. (32) is naturally applicable to other values of $N$ as well. Figure 1(b) shows that all possible column matrices $\boldsymbol{J}$ are really considered in the 32660 runs because the scalar product gets all values between -1 and +1 . In other words, this means that $J$ is rotated through the unit circle in the plane de ned by the requirement that $J_{1}+J_{2}+J_{3}=0$. (Referring to the comment about the units at the end of Section 3.1, we 
(a)

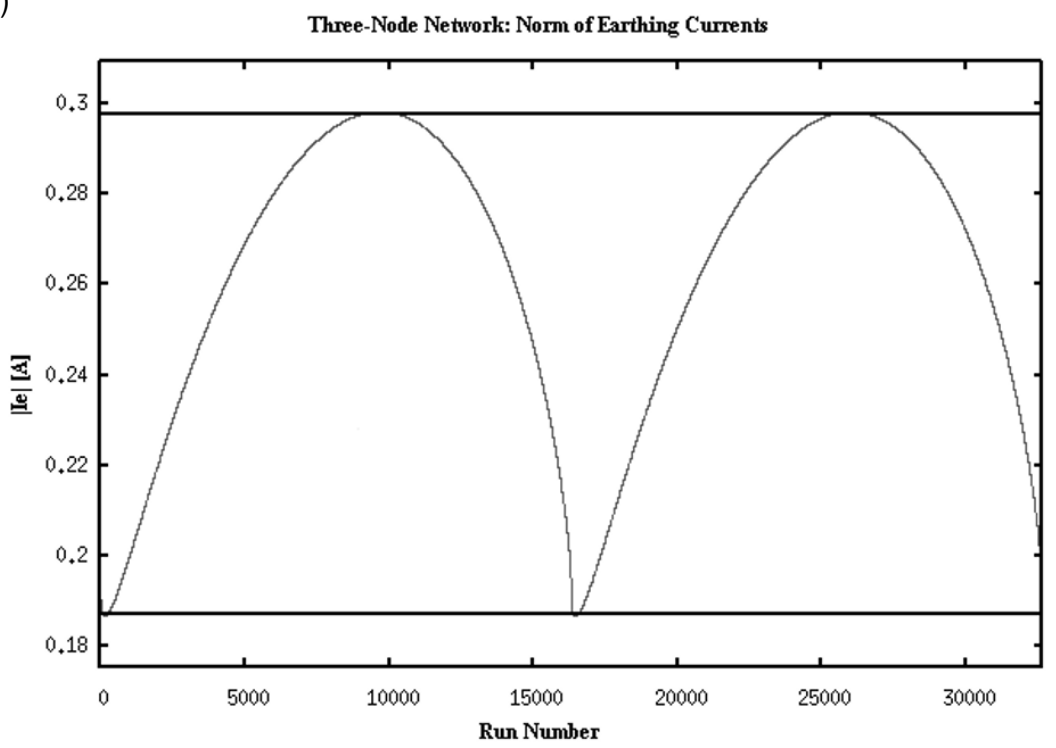

(b)

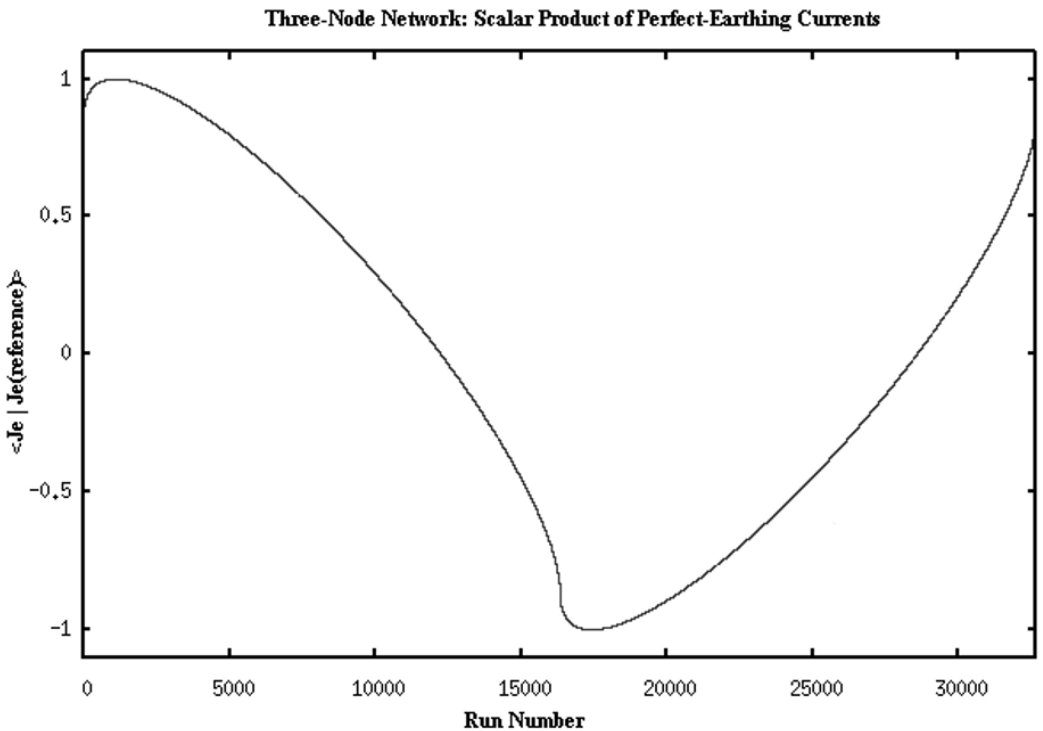

Fig. 1. (a) Norm of the earthing GIC column matrix for a three-node network when the pe current column matrix is normalised to unity. The earthing resistances of the nodes are $S_{1}=1.00 \Omega, S_{2}=0.64 \Omega$ and $S_{3}=0.88 \Omega$, and the line resistances are $R_{1}$ (line 1-2) $=0.50 \Omega, R_{2}($ line $2-3)=1.20$ $\Omega$ and $R_{3}$ (line 3-1) $=0.87 \Omega$. Altogether 32660 runs of the calculation program are included by changing the pe current column matrix slightly and systematically between the runs and requiring that the sum of the elements of the pe matrix is zero. The horizontal lines show two of the eigenvalues of the transfer matrix between pe currents and earthing GIC (while the third eigenvalue equals one). (b) Scalar product between the pe current column matrices used in Fig. 1(a) for the 32660 runs and a fixed reference column matrix normalised to unity and having elements whose sum is zero.

should actually say that $\boldsymbol{J}$ and $\boldsymbol{J}_{\text {ref }}$ are normalised to $1 \mathrm{~A}$ making $\left[\mathrm{A}^{2}\right]$ be the unit of the scalar product.)

The eigenvalues of $C$ are $\lambda_{1}=1, \lambda_{2}=0.1869$ and $\lambda_{3}=0.2978$, the two latter of which are indicated by the horizontal lines in Fig. 1(a). We see that the norm of the earthing currents mostly lies between $\lambda_{2}$ and $\lambda_{3}$ but not always. In Fig. 1(a), the minimum and maximum of $|\boldsymbol{I}|$ are 0.1867 and 0.2982 , respectively. In practice, there is no difference between $\lambda_{2}$ and $\min (|\boldsymbol{I}|)$ and between $\lambda_{3}$ and $\max (|\boldsymbol{I}|)$. However, the positive differences $\lambda_{2}-\min (|\boldsymbol{I}|)$ and $\max (|\boldsymbol{I}|)-\lambda_{3}$ are larger than the possible numerical inaccuracy in the computations. In the calculations presented in Fig. 1(a), altogether $0.79 \%$ and $6.66 \%$ of the $|\boldsymbol{I}|$ values are below $\lambda_{2}$ and above $\lambda_{3}$, respectively. Thinking about practical GIC research, $|\boldsymbol{I}|$ gives a kind of an overall average impact of GIC on transformers, and the present calculation indicates that $\lambda_{2}$ and $\lambda_{3}$ determine the lower and upper limit of this impact. In this connection, it is necessary to emphasize that because $\boldsymbol{J}$ is normalised to unity the values of $|\boldsymbol{I}|$ are quite small in Fig. 1(a). In a practical GIC case, $|\boldsymbol{J}|$ is much larger than $1 \mathrm{~A}$, and $\lambda_{2}|\boldsymbol{J}|$ and $\lambda_{3}|\boldsymbol{J}|$ give the limits for $|\boldsymbol{I}|$. This comment also concerns the numerical calculations presented below in this paper.

Figures 2(a) and 2(b) show the same quantities as Figs. 1(a) and 1(b), respectively. Significant differences are, however, involved in the assumptions involved in Figs. 2(a) and 2(b) compared to Figs. 1(a) and 1(b). The resistances are now chosen by a randomisation procedure in reasonable ranges, which has resulted in the values $S_{1}=0.96 \Omega$, $S_{2}=1.10 \Omega, S_{3}=0.80 \Omega, R_{1}=1.62 \Omega, R_{2}=0.97 \Omega$ and 
(a)

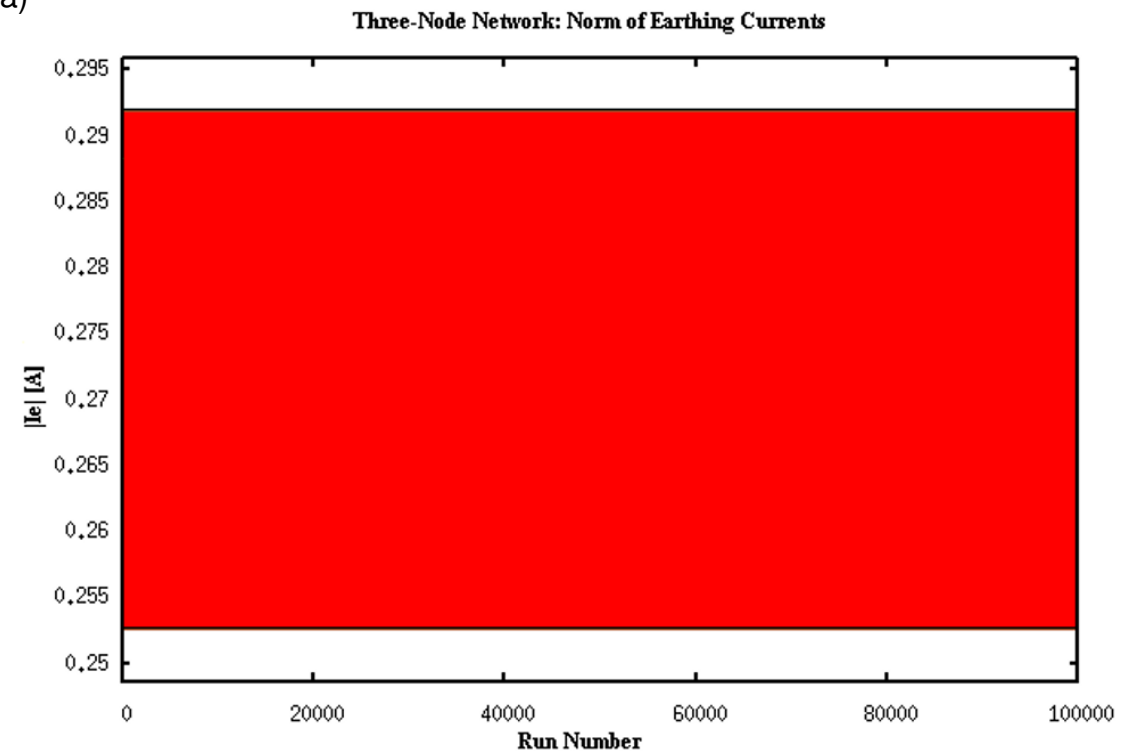

(b)

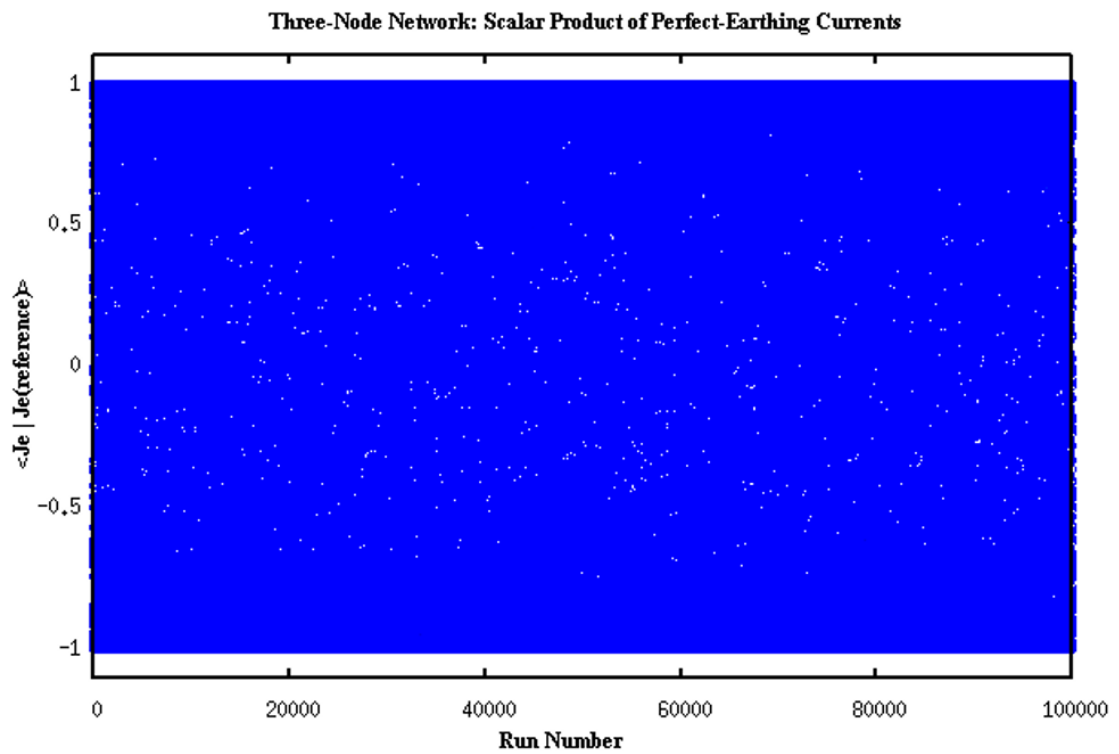

Fig. 2. (a) Norm of the earthing GIC column matrix for a three-node network when the pe current column matrix is normalised to unity. The earthing resistances of the nodes are $S_{1}=0.96 \Omega, S_{2}=1.10 \Omega$ and $S_{3}=0.80 \Omega$, and the line resistances are $R_{1}$ (line 1-2) $=1.62 \Omega, R_{2}($ line $2-3)=0.97 \Omega$ and $R_{3}$ (line 3-1) $=0.82 \Omega$. All these resistance values are chosen randomly in reasonable ranges. The pe current column matrix is also determined randomly for every run of the calculation program requiring that the sum of the elements of the pe matrix is zero. The total number of the runs is 100000. The horizontal lines show two of the eigenvalues of the transfer matrix between pe currents and earthing GIC (while the third eigenvalue equals one). It is seen that the values of the norm of the earthing GIC cover the range between the two eigenvalues very accurately. (b) Scalar product between the pe current column matrices used in Fig. 2(a) for the 100000 runs and a fixed reference column matrix normalised to unity and having elements whose sum is zero. It is seen that the scalar product values cover the whole range from -1 to +1 very well.

$R_{3}=0.82 \Omega$ to be used for Figs. 2(a) and 2(b). The pe currents $J_{1}, J_{2}$ and $J_{3}$ are also determined randomly for every run of the calculation program with the only constraints that they satisfy Eq. (6) and $\boldsymbol{J}$ is normalised to unity. The total number of the program runs is 100000 . From Fig. 2(b) we notice that all scalar product values between -1 and +1 are encountered, which implies that all possible column matrices $\boldsymbol{J}$ are included in the computations. Looking at Fig. 2(b) carefully, it can be seen that values near \pm 1 are a little better represented than values closer to zero. This is clearly a consequence of the fact that the cosine function, which is present in the scalar product, varies more slowly around \pm 1 that at zero.
The eigenvalues $\lambda_{2}=0.2525524$ and $\lambda_{3}=0.2919066$ of $\boldsymbol{C}$ are shown by the horizontal lines in Fig. 2(a), and we see that the values of the norm $|\boldsymbol{I}|$ cover the area between $\lambda_{2}$ and $\lambda_{3}$ very well. However, if the values are considered exactly, we can again note that $|\boldsymbol{I}|$ can be slightly lower than $\lambda_{2}$ or slightly larger than $\lambda_{3}$ since $\min (|\boldsymbol{I}|)$ and $\max (|\boldsymbol{I}|)$ are 0.2525465 and 0.2919134 , respectively. Similarly to Fig. 1(a), the positive differences $\lambda_{2}-\min (|\boldsymbol{I}|)$ and $\max (|\boldsymbol{I}|)-\lambda_{3}$ are, on one hand, insignificant in practice, but on the other hand, cannot be explained by numerical inaccuracies in the computations. Anyway, Fig. 2(a) supports the conclusion already drawn from Fig. 1(a) that $\lambda_{2}$ and $\lambda_{3}$ express the lower and upper limit of the practical GIC impact 
on the network.

Choosing the resistances to be $S_{1}=0.50 \Omega, S_{2}=7.50$ $\Omega, S_{3}=0.75 \Omega, R_{1}=0.80 \Omega, R_{2}=1.20 \Omega$ and $R_{3}=0.08$ $\Omega$, we have the resonance case discussed in Section 3.2.2 since Eq. (28) are satis ed (with the products equalling 0.60 $\Omega^{2}$ ). According to the theory, the eigenvalues $\lambda_{2}$ and $\lambda_{3}$ of $\boldsymbol{C}$ are the same, and all column matrices $\boldsymbol{J}=\left(\begin{array}{l}J_{1} \\ J_{2} \\ J_{3}\end{array}\right)$ for which $J_{1}+J_{2}+J_{3}=0$ are corresponding eigenvectors. The value of $\lambda_{2}=\lambda_{3}$ can be calculated from Eq. (31) to obtain 0.057971 . The fact that $\lambda_{2}=\lambda_{3}$ is much smaller than the values of $\lambda_{2}$ and $\lambda_{3}$ in the cases investigated in Figs. 1(a) and 2(a) obviously results from the large resistance $S_{2}$. Decreasing $S_{2}$ by a factor of 4 and increasing $R_{3}$ by the same factor, i.e. $S_{2}=1.875 \Omega, R_{3}=0.32 \Omega$, so that Eq. (28) still hold true, give a clearly larger value $\lambda_{2}=\lambda_{3}=0.18079$.

\subsection{Finnish $400 \mathrm{kV}$ test model}

Pirjola (2009) introduces an old version of the Finnish $400 \mathrm{kV}$ power network valid in 1978 to 1979 as a test model for GIC calculation algorithms and programs. The geographical structure of today's $400 \mathrm{kV}$ grid in Finland is quite similar to the particular old version though not the same. Regarding GIC, an essential difference is produced by the installation of series capacitors in north-south lines (Elovaara, 2007). They block the ow of the dc-like GIC, which means that the Finnish $400 \mathrm{kV}$ network consists of two separate parts as concerns GIC nowadays. (It should be noted that the reasons for the use of series capacitors are other than GIC mitigation.)

The Finnish $400 \mathrm{kV}$ test model has 17 stations and 19 transmission lines, thus being complex enough to enable realistic GIC calculations but not too large to unnecessarily hamper the computations. The Cartesian at-Earth coordinates and (total) earthing resistances of the stations and the line resistances included in the test model are provided by Pirjola (2009). A special feature is that the earthing resistances of the two northwestern end stations are zero to account for the galvanic connection of the grid to the Swedish $400 \mathrm{kV}$ network. It is additionally assumed that the stations are distant enough, so the earthing impedance matrix is diagonal. As Pirjola (2008) points out, it is not an essential limitation in practice. For reference information to the users of the test model, Pirjola (2009) also presents the values of GIC owing to (or from) the Earth at the stations and of GIC in the transmission lines when the test model is impacted by a northward or an eastward uniform geoelectric eld of $1 \mathrm{~V} / \mathrm{km}$.

Figures 3(a) and 3(b) are similar to Figs. 2(a) and 2(b), except that the resistances are not chosen randomly but the correct test model values are used. A great difference is also, of course, that $C$ is a $17 \times 17$ matrix leading to 17 eigenvalues and implying that analytic solutions are impossible in practice. Figure 2(a) presents the norm of $\boldsymbol{I}=\boldsymbol{C J}$ (Eq. (13)) when the $17 \times 1$ pe column matrix is determined in a random way, with the only constraints that $|\boldsymbol{J}|=1$ and Eq. (6) is satis ed, for each of the 100000 calculation program runs. The horizontal lines in the gure indicate the eigenvalues. They are seen to satisfy the inequalities (12). The smallest and the largest $(\neq 1)$ eigenvalue are plotted with thicker lines. We see that these extreme values clearly restrict the range of $|\boldsymbol{I}|$. Actually $|\boldsymbol{I}|$ never reaches a value that is even very close to either of the extremes in the 100000 runs performed. Looking at Fig. 3(b), we see that the random choices made in the runs do not cover all possible $\boldsymbol{J}$ matrices because the scalar product values are concentrated in the range from about -0.6 to about +0.6 and never achieve values around \pm 1 . Mathematically, this may be understood by noting that we are operating in a 16-dimensional vector space. (One dimension of the 17-dimensional space is removed by the requirement that Eq. (6) is satis ed.) In Figs. 2(a) and 2(b), in which the vector space is two-dimensional, 100000 runs of the program are suf cient, but we would need a much larger number of runs to be able to fully investigate $|\boldsymbol{I}|$ associated with the test model in question. Considering the Manitoba high-voltage network in Canada in a similar way (not shown here), we see that both the $|\boldsymbol{I}|$ values and the scalar products are concentrated in even smaller ranges as the number of nodes is about ten times larger than in the test model.

An interesting detail concerning the test model is that the eigenvalue $\lambda_{1}=1$ is a double value, so it has two independent eigenvectors. Looking at the issue more carefully, we see that the eigenvectors correspond to situations where the pe current has a non-zero value at one of the two northwestern zero-resistance stations and is zero elsewhere. Considering such a case in terms of external injection of the pe currents as mentioned in Section 2, the situation simply means that a current injected at a zero-resistance station directly ows into the Earth and nothing happens at other sites of the network. Thus, this is in accordance with what is mentioned about the pe currents associated with the eigenvalue equal to one in Section 3.1. It is easy to understand physically but does not have any practical interest or importance.

Besides Manitoba (Canada), similar calculations have also been performed for the high-voltage power networks in Brazil, China (ultra-high-voltage) and Southern Sweden. In every case, the inequalities (12) are valid as expected. Also otherwise, the results are comparable to those for the Finnish $400 \mathrm{kV}$ test model. These additional results support the above-mentioned conclusion valid for the Manitoba case that an increase of the number of nodes makes the plots corresponding to Figs. 3(a) and 3(b) concentrate in smaller ranges when the calculation program is run (only) 100000 times. A detail worth noting as well is that, similarly to the three-node network and the Finnish $400 \mathrm{kV}$ test model, the value $\lambda=1$ (with a high accuracy) seems to be included in the set of eigenvalues in each calculation at least once. Finally, we emphasise that an earthing impedance matrix with non-zero off-diagonal elements (which is not the case for the three-node and test model cases) is included in the Manitoba and Southern Sweden computations. Because the conclusions are similar from all calculations, we thus obtain additional support to the fact that assuming the earthing impedance matrix to be diagonal is not a serious restriction in practice.

\section{Concluding Remarks}

Geomagnetically induced currents (GIC) owing in power networks due to space weather can be calculated by 
(a)

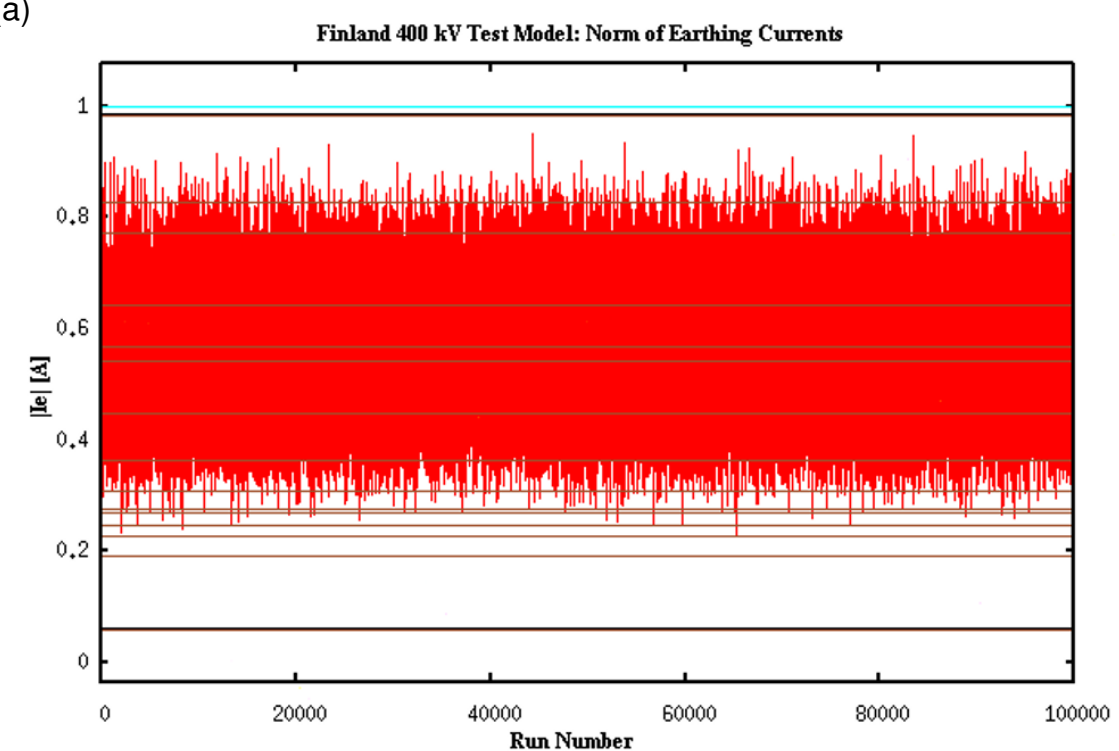

(b)

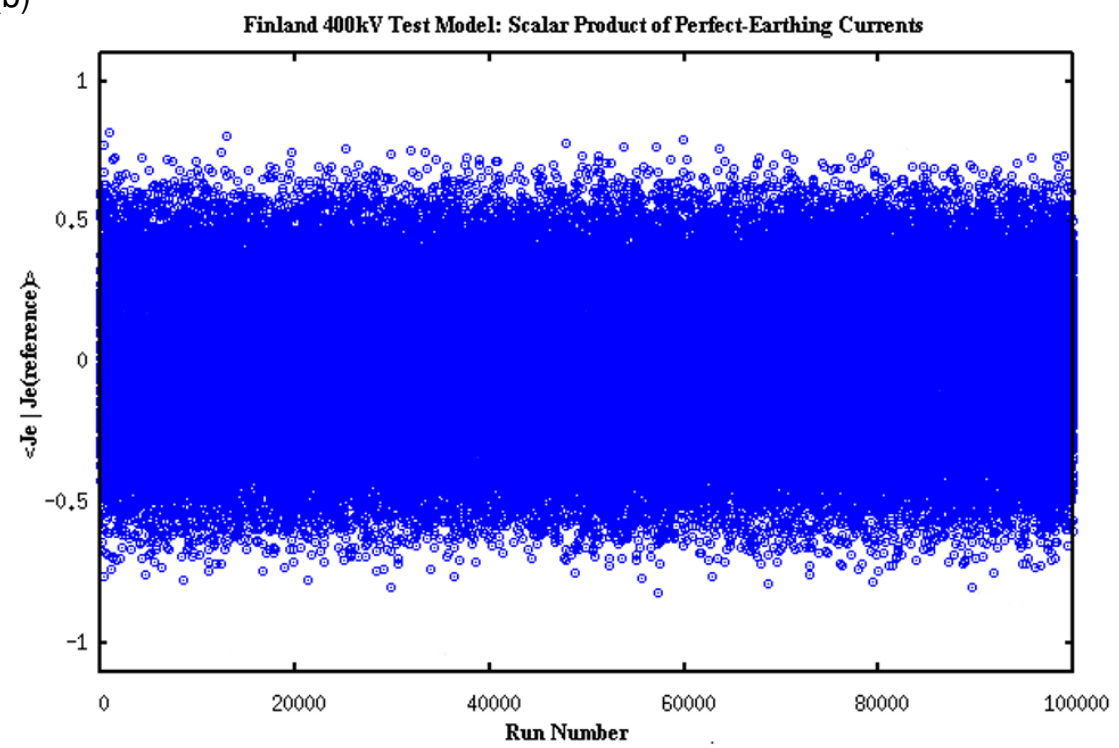

Fig. 3. (a) Norm of the earthing GIC column matrix for the Finnish $400 \mathrm{kV}$ test model (see Pirjola, 2009) when the pe current column matrix is normalised to unity. The pe current column matrix is determined randomly for every run of the calculation program requiring that the sum of the elements of the pe matrix is zero. The total number of the runs is 100000 . The horizontal lines show the eigenvalues of the transfer matrix between $p e$ currents and earthing GIC. It is seen that the values of the norm of the earthing GIC are clearly between the smallest eigenvalue and the largest $(\neq 1)$ eigenvalue, both of which are plotted with thicker lines. (b) Scalar product between the pe current column matrices used in Fig. 3(a) for the 100000 runs and a fixed reference column matrix normalised to unity and having elements whose sum is zero. It is seen that the scalar product values do not cover the range from -1 to +1 , which means that the 100000 runs do not include all possible pe current matrices.

using convenient matrix formulas whose input data consist of the horizontal geoelectric field at the Earth's surface and of the network resistances and topology. This paper is focussed on the transfer matrix between the so-called "perfect-earthing" ( $p e$ ) currents and GIC flowing between the Earth and the network. The pe currents depend on the geovoltages impacting the transmission lines and produced by the geoelectric field. The transfer matrix depends on the network resistances and topology in terms of an earthing impedance matrix and a network admittance matrix.

This paper provides a novel approach to the transfer matrix by considering its eigenvalues and eigenvectors. It is shown that either an eigenvalue equals one or the corresponding eigenvector satisfies the condition that the sum of the $p e$ currents included in the particular eigenvector is zero, which implies that the amounts of pe currents flowing to and from the network are equal. The situation in which the eigenvalue equals one seems to be unimportant regarding practical GIC applications.

By physical arguments, we conclude in this paper that all eigenvalues of the transfer matrix are non-negative and less than or equal to one. This statement is proved also mathematically in the case of a three-node network, which is simple enough to enable analytic calculations related to eigenvalues and eigenvectors of the transfer matrix. It also seems evident that some conclusions drawn from the three-node case might be generalised to larger networks as well. In this paper, numerical computations that concern the Finnish 400 
$\mathrm{kV}$ GIC test model also support that the eigenvalues lie between zero and one. The same eigenvalue range is indicated by numerical calculations for other power grids as well.

Special attention is paid to the norm (= square root of the sum of the squares) of the earthing GIC owing between the Earth and the network. It is a quantity that gives an overall idea of the possibility of adverse impacts of GIC on a power grid since it is associated with GIC owing through transformers. If the pe current matrix is adjusted to have the norm equal to one, the norm of the earthing GIC seems to have the lower and upper limits equal to the smallest and largest (but $\neq 1$ ) eigenvalue of the transfer matrix. This is an observation valid in practical GIC studies but not exactly. Without the normalisation of the pe current matrix to one, the lower and upper limits equal the smallest and largest eigenvalue multiplied by the norm of the pe currents.

Acknowledgments. The author wishes to thank Dr. Ari Viljanen (Finnish Meteorological Institute) for many useful discussions on the topic of this paper. Thanks also go to all colleagues in the collaboration concerning the Finnish, Swedish, Manitoba, Brazilian and Chinese power networks discussed in this paper.

\section{References}

Bolduc, L., GIC observations and studies in the Hydro-Québec power system, J. Atmos. Sol.-Terr. Phys., 64(16), 1793-1802, 2002.

Boteler, D. H. and R. J. Pirjola, Modelling geomagnetically induced currents produced by realistic and uniform electric elds, IEEE Trans. Power Delivery, 13(4), 1303-1308, 1998.

Boteler, D. H., R. J. Pirjola, and H. Nevanlinna, The effects of geomagnetic disturbances on electrical systems at the earth's surface, Adv. Space Res.,
22(1), 17-27, 1998.

Elovaara, J., Finnish experiences with grid effects of GIC's, in Space Weather, Research towards Applications in Europe, edited by J. Lilensten, Chapter 5.4, 311-326, Astrophysics and Space Science Library, 344, ESA, COST 724, Springer, 2007.

Kappenman, J. G., Geomagnetic disturbances and impacts upon power system operation, in The Electric Power Engineering Handbook, 2nd edition, edited by L. L. Grigsby, Chapter 16, 16-1-16-22, CRC Press/IEEE Press, 2007.

Lanzerotti, L. J., D. J. Thomson, and C. G. Maclennan, Engineering issues in space weather, in Modern Radio Science 1999, edited by M. A. Stuchly, 25-50, International Union of Radio Science (URSI), Oxford University Press, 1999.

Lehtinen, M. and R. Pirjola, Currents produced in earthed conductor networks by geomagnetically-induced electric elds, Ann. Geophys., 3(4), 479-484, 1985.

Molinski, T. S., Why utilities respect geomagnetically induced currents, $J$. Atmos. Sol.-Terr. Phys., 64(16), 1765-1778, 2002.

Pirjola, R., Review on the calculation of surface electric and magnetic elds and of geomagnetically induced currents in ground-based technological systems, Surv. Geophys., 23(1), 71-90, 2002.

Pirjola, R., Effects of interactions between stations on the calculation of geomagnetically induced currents in an electric power transmission system, Earth Planets Space, 60(7), 743-751, 2008.

Pirjola, R., Properties of matrices included in the calculation of geomagnetically induced currents (GICs) in power systems and introduction of a test model for GIC computation algorithms, Earth Planets Space, 61(2), 263-272, 2009.

Viljanen, A., A. Pulkkinen, O. Amm, R. Pirjola, T. Korja, and BEAR Working Group, Fast computation of the geoelectric eld using the method of elementary current systems and planar Earth models, Ann. Geophys., 22(1), 101-113, 2004.

R. Pirjola (e-mail: risto.pirjola@fmi. ) 